\title{
Electronic structure of $\mathrm{Ba}(\mathrm{Fe}, \mathrm{Ru})_{2} \mathrm{As}_{2}$ and $\mathrm{Sr}(\mathrm{Fe}, \mathrm{Ir})_{2} \mathrm{As}_{2}$ alloys
}

\author{
Lijun Zhang and D.J. Singh \\ Materials Science and Technology Division, Oak Ridge National Laboratory, Oak Ridge, Tennessee 37831-6114
}

(Dated: November 29, 2018)

\begin{abstract}
The electronic structures of $\mathrm{Ba}(\mathrm{Fe}, \mathrm{Ru})_{2} \mathrm{As}_{2}$ and $\mathrm{Sr}(\mathrm{Fe}, \mathrm{Ir})_{2} \mathrm{As}_{2}$ are investigated using density functional calculations. We find that these systems behave as coherent alloys from the electronic structure point of view. In particular, the isoelectronic substitution of Fe by Ru does not provide doping, but rather suppresses the spin density wave characteristic of the pure Fe compound by a reduction in the Stoner enhancement and an increase in the band width due hybridization involving Ru. The electronic structure near the Fermi level otherwise remains quite similar to that of $\mathrm{BaFe}_{2} \mathrm{As}_{2}$. The behavior of the Ir alloy is similar, except that in this case there is additional electron doping.
\end{abstract}

PACS numbers: 74.25.Jb,71.20.Be

\section{INTRODUCTION}

The discovery of unconventional superconductivity in proximity to magnetism for layered Fe-based materials $1,2,3,4,5,6$ has led to considerable interest in both establishing the interplay between magnetic order and superconducting state, and searching for effective ways of tuning them. The phase diagrams typically show a spin density wave (SDW) that competes with superconductivity, i.e. superconductivity generally appears when the SDW is suppressed either by doping with electrons or holes, which reduces the nesting by making the electron and hole Fermi surfaces mismatched or by pressure, ${ }^{7}$ which increases hybridization and broadens the bands again working against nesting.

A remarkable feature of these compounds is that, in contrast to the cuprates, they may be doped into a superconducting state by substitutions on the Fe-site, e.g. in $\mathrm{Ba}(\mathrm{Fe}, \mathrm{Co})_{2} \mathrm{As}_{2}$ and $\mathrm{Ba}\left(\mathrm{Fe}, \mathrm{Ni}_{2}\right)_{2} \mathrm{As}_{2} \underline{\underline{8}, \underline{9}}$ In these alloys the electronic structure remains very similar to that of the parent $\mathrm{Ba}(\mathrm{Sr}) \mathrm{Fe}_{2} \mathrm{As}_{2}$, but the Fermi level is shifted upwards corresponding to the increased electron count. Thus one difference from cuprates is that substitution of $\mathrm{Co}$ and $\mathrm{Ni}$ lead to the formation of a coherent alloy electronic structure rather than the introduction of localized states associated with those ions. Recently, it has been shown that in addition to the $3 d$ dopants, Co and $\mathrm{Ni}$, superconductivity can be induced by alloying with some $4 d$ and $5 d$ elements, including $\mathrm{Ru}, \mathrm{Rh}, \mathrm{Ir}$ and $\operatorname{Pd} \cdot 10,11,12,13,14,15$

Transition elements in the $4 d$ and $5 d$ rows differ from their $3 d$ counterparts in several respects. Since $4 d$ and $5 d$ orbitals are much more extended than the $3 d$ orbitals there is a greater tendency towards covalency both with ligands (e.g. As) and also in stronger metal - metal $d$ bonds. For example, mid-5d transition elements have some of the highest melting points of any material (the melting points of Ir and Ru are $2739 \mathrm{~K}$ and $2607 \mathrm{~K}$, respectively, as compared to $1811 \mathrm{~K}$ for $\mathrm{Fe}$ and $3695 \mathrm{~K}$ for $\mathrm{W}$ ), and compounds of these elements are often extremely hard. Thus one may expect broader bands and more hybridization with As in the alloys with these elements. Secondly, again because of the more extended
$4 d$ and $5 d$ orbitals, the Hund's coupling on these atoms is weaker than on $3 d$ atoms, which works against magnetism and is reflected in lower values of the Stoner parameter for $4 d$ and $5 d$ elements and compounds. Finally, the larger orbitals lead to a tendency for higher valence states in the $4 d$ and $5 d$ series, so that $\mathrm{Ru}^{4+}$ and $\mathrm{Ru}^{5+}$ compounds are more stable and more common than the corresponding Fe compounds.

Returning to superconductivity in alloys of $\mathrm{BaFe}_{2} \mathrm{As}_{2}$ with $4 d$ and $5 d$ elements, the case of $\mathrm{Ru}$ is particularly interesting because $\mathrm{Ru}$ is isoelectronic with $\mathrm{Fe}$ and therefore it may or may not serve as a dopant. Transition temperatures up to $T_{c} \sim 21 \mathrm{~K}$ may obtained with substantial nominal $\mathrm{Ru}$ content in $\mathrm{Ba}(\mathrm{Sr}) \mathrm{Fe}_{2-x} \mathrm{Ru}_{x} \mathrm{As}_{2}, x_{\text {nom }} \sim 0.7$ although $\mathrm{Ba}(\mathrm{Sr}) \mathrm{Ru}_{2} \mathrm{As}_{2}$ shows neither magnetic order nor superconductivity $\underline{\underline{16}}$ One possibility is that Ru serves as a dopant, supplying carriers to the Fe planes, $\stackrel{10,12}{\text { For }}$ example, $\mathrm{Ru}$ might occur as $\mathrm{Ru}^{4+}$, in which case empty localized $d$ states associated with $\mathrm{Ru}$ atoms would occur above the Fermi energy, while the Fe derived valence bands would show higher filling reflecting electron doping by two carriers per Ru. Another possibility is that alloy shows a coherent electronic structure that is distorted from the pure Fe compound, but does not show additional states, similar to the Co and Ni doped materials. In this case, the effect of $\mathrm{Ru}$ could be similar to that of pressure, broadening the bands, increasing hybridization and/or lowering the effective Hund's coupling and thereby destroying the SDW in favor of superconductivity.

Here, we report density functional studies of the electronic structure of $\mathrm{BaFe}_{2-x} \mathrm{Ru}_{x} \mathrm{As}_{2}$. It is found that the substitution of $\mathrm{Fe}$ with $\mathrm{Ru}$ gives a quite similar electronic structure to that of $\mathrm{BaFe}_{2} \mathrm{As}_{2}$ near the Fermi level $E_{F}$. Importantly, this substitution does not result in doping since we find no additional flat bands reflecting $\mathrm{Ru}$ states in supercell calculations and consequently the exact Luttinger's theorem compensation of electron and hole surfaces is maintained in the same way as in the pure compound without the SDW. Thus, Ru substitution does not introduce additional electrons to this system. Instead the SDW magnetic order is suppressed by the decreasing Stoner term and increased hybridization. We also per- 
formed the calculation for $5 d$ transition metal, Ir alloyed $\mathrm{SrFe}_{1-x} \mathrm{Ir}_{x} \mathrm{As}_{2}$, which was reported to superconduct with $T_{c} \sim 22 \mathrm{~K} .15$ We also find an electronic structure characteristic of a coherent alloy for this non-isoelectronic, Co column transition metal. We find electron doping as expected with additional one electron per Ir, which cooperates with the reduction of Hund's coupling to destabilize the SDW order.

\section{FIRST PRINCIPLES CALCULATIONS}

Our first principles electronic structure calculations were performed within the generalized gradient approximation (GGA) of Perdew, Burke, and Ernzerhof $(\mathrm{PBE}) \stackrel{17}{\stackrel{1}{1}}$ using the general potential linearized augmented planewave (LAPW) method, with the augmented planewave plus local orbital implementation. $\underline{18,19,20}$ LAPW spheres of radii $2.3 a_{0}$ for $\mathrm{Ba}$, Ir and $2.1 a_{0}$ for $\mathrm{Fe}, \mathrm{As}, \mathrm{Ru}$ were employed. We used well converged basis sets determined by $R_{\min } K_{\max }=8.5$, where $R_{\min }$ is the radius of the smallest LAPW sphere and $K_{\max }$ corresponds to the planewave cutoff for the interstitial region. We included relativity at the scalar relativistic level. Local orbitals were included to accurately treat the semi-core states. The Brillouin zone sampling for self-consistent calculations was done using the special k-point method, with a $24 \times 24 \times 24$ grid for body-centered $\mathrm{ThCr}_{2} \mathrm{Si}_{2}$ structure and a $21 \times 21 \times 9$ grid for the quadrupled tetragonal supercells with partial $\mathrm{Ru}$ and Ir substitution (see below).

\section{RUTHENIUM SUBSTITUTION}

We first calculated the electronic structure of completely $\mathrm{Ru}$ substituted non-superconducting $\mathrm{BaRu}_{2} \mathrm{As}_{2}$ to check whether additional bands or significant changes in the band shapes are introduced by Ru. The experimental tetragonal lattice constant $a=4.152 \AA$ and $c=$ $12.238 \AA^{21}$ were employed and the internal As coordinate $z_{\mathrm{As}}$ were optimized by total energy minimization as 0.3528. For this material, we checked for but did not find magnetic order of either ferromagnetic or antiferromagnetic character, consistent with recent experiments $\underline{16}$ Fig. 1 shows the calculated band structure, in comparison with that of non-spin-polarized $\mathrm{BaFe}_{2} \mathrm{As}_{2}$ as obtained with the same approach. As may be seen, the general features of band structures around $E_{F}$ for two compounds show quite close similarity and no new bands appear in $\mathrm{BaRu}_{2} \mathrm{As}_{2}$. In particular, there are similar compensating electron Fermi surface sections at the zone corner and hole sections at the zone center ( $\Gamma$ - $\mathrm{Z}$ direction). Especially at the zone corner, there is a very strong similarity of the electron cylinders in the two compounds. However, for $\mathrm{BaRu}_{2} \mathrm{As}_{2}$ we do find a somewhat different structure of the hole Fermi surfaces, which are centered near $\mathrm{Z}$ as compared to $\mathrm{BaFe}_{2} \mathrm{As}_{2}$ where hole bands exist
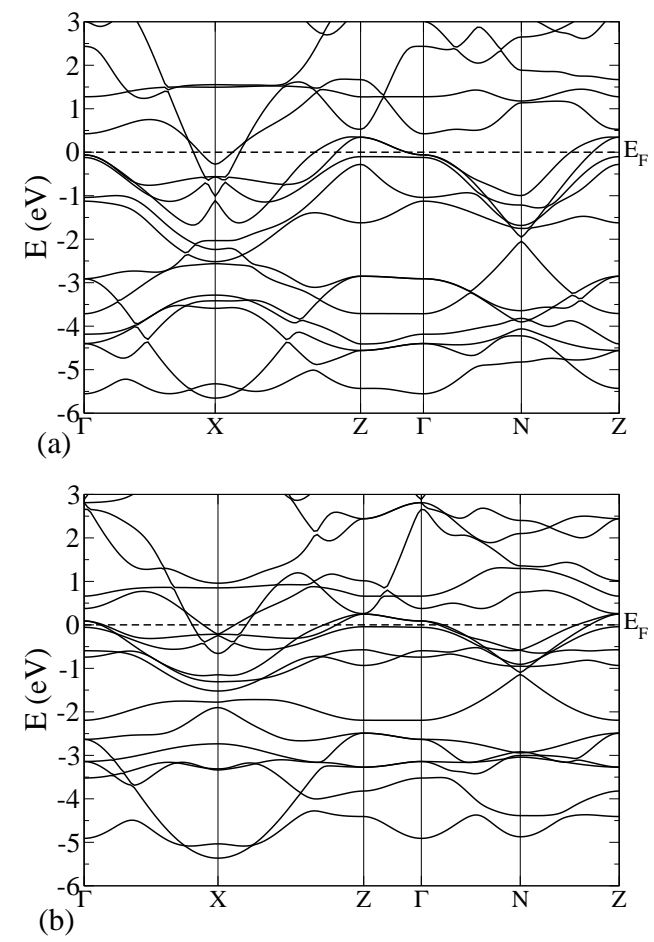

FIG. 1: Calculated electronic band structure of (a) $\mathrm{BaRu}_{2} \mathrm{As}_{2}$ with the relaxed $z_{\mathrm{As}}$, comparing with that of (b) $\mathrm{BaFe}_{2} \mathrm{As}_{2}$. The bands are plotted along high-symmetry directions in the body-centered tetragonal Brillouin zone, and $X$ point corresponds to the zone corner $M$ point in the tetragonal Brillouin zone. For $\mathrm{BaFe}_{2} \mathrm{As}_{2}$, we used the experimental lattice parameter $a=3.9625 \AA$ and $c=13.0168 \AA$ (Ref. 24) and non-spin-polarized GGA optimized As height $z_{\mathrm{As}}=0.344$.

along the $\Gamma-\mathrm{Z}$ direction. This yields a closed hole Fermi surface in the $k_{z}$ direction in $\mathrm{BaRu}_{2} \mathrm{As}_{2}$. This more 3D shape is suggestive of more $\mathrm{Ru}-\mathrm{As}$ hybridization, which is also seen in the projections of the electronic density of states (DOS). In any case, the position of $E_{F}$ with respect to the compensating position between the hole and electron band edges is maintained similar to nonspin-polarized $\mathrm{BaFe}_{2} \mathrm{As}_{2}$. This is different from the nonisoelectronic compounds, $\mathrm{BaCO}_{2} \mathrm{As}_{2} \stackrel{22}{2}$ and $\mathrm{BaNi}_{2} \mathrm{As}_{2}, \stackrel{23}{=}$ which also show rather similar electronic structures to the Fe compound, but with the $E_{F}$ shifted to higher energy corresonding to the higher electron count. This shift of the Fermi energy results in dramatically different physical properties.

We used supercell calculations to explicitly study the effects of partial Ru and Ir substitution. For this purpose we used a $\sqrt{2} \mathrm{x} \sqrt{2}$ doubling in plane, and also doubled the cell along the $c$-axis by going to the conventional tetragonal cell as opposed to the body centered cell. This leads to a quadrupling of the $\mathrm{ThCr}_{2} \mathrm{Si}_{2}$ structure cell. We then replaced one $\mathrm{Fe}$ by $\mathrm{Ru}$ or $\mathrm{Ir}$ in each plane. This corresponds to a composition of $\mathrm{BaFe}_{1.5} \mathrm{Ru}_{0.5} \mathrm{As}_{2}$ (nominal $\mathrm{Ba}_{4} \mathrm{Fe}_{6} \mathrm{Ru}_{2} \mathrm{As}_{8}$ ) or $\mathrm{SrFe}_{1.5} \mathrm{Ir}_{0.5} \mathrm{As}_{2}$. For $\mathrm{Ba}_{4} \mathrm{Fe}_{6} \mathrm{Ru}_{2} \mathrm{As}_{8}$ we did calculations for three different arrangements of the 


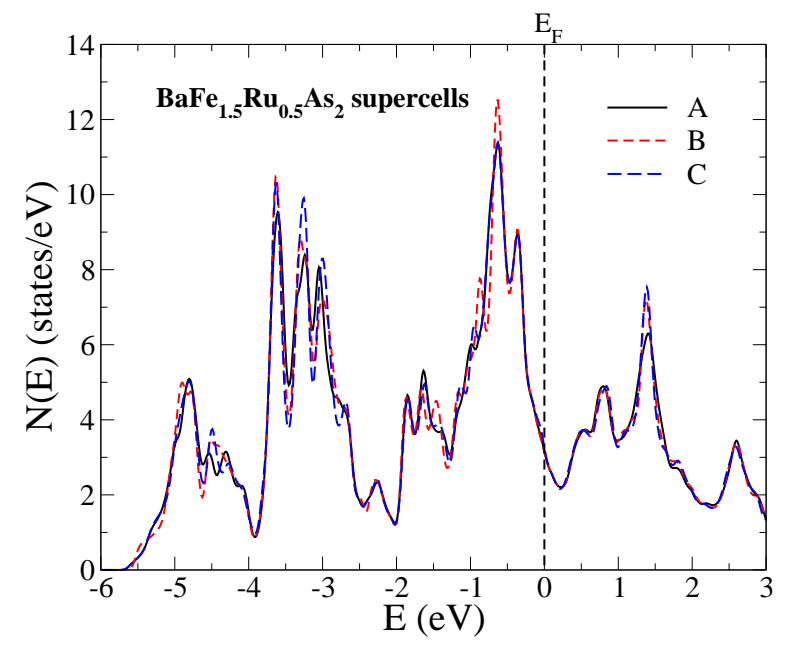

FIG. 2: (Color online) Comparison of the calculated electronic DOS for the three $\mathrm{BaFe}_{1.5} \mathrm{Ru}_{0.5} \mathrm{As}_{2}$ supercells, $A, B$ and $C$ as described in the text.

$\mathrm{Ru}$. These were with the two Ru directly on top of each other $(A)$, shifted by one Fe-Fe distance $(B)$ and shifted by one lattice parameter, $a(C)$. The tetragonal lattice parameter for $\mathrm{BaFe}_{1.5} \mathrm{Ru}_{0.5} \mathrm{As}_{2}, a=3.98 \AA$ and $c=12.95$ $\AA$ were taken from Ref. 10 and the internal coordinates were optimized by energy minimization. As shown in Fig. 2, the electronic structures for these three supercells are very similar. The values at the Fermi energy for the three cells are $N\left(E_{F}\right)=3.17(A), N\left(E_{F}\right)=3.14(B)$, and $N\left(E_{F}\right)=3.17(C)$. In the following we focus on results for supercell $A$, since the same conclusions would be drawn based on the others.

It is found that, as the result of larger size for $\mathrm{Ru}^{2+}$, apart from expanding distances within $\mathrm{Fe}-\mathrm{Ru}$ planes, 10 the $\mathrm{Ru}$-As distance $(2.41 \AA)$ is also slightly larger than the Fe-As distances $(2.30 \AA-2.35 \AA)$, and accordingly the $\mathrm{Ba}$ layers are also distorted. The calculated DOS is shown in Fig. 3 in comparison with that of $\mathrm{BaFe}_{2} \mathrm{As}_{2}$. We did not find any additional peak associated with extra flat bands introduced by $\mathrm{Ru}$ substitution. This and the shape of the DOS reflecting bands of mixed Fe and $\mathrm{Ru}$ character, as opposed to separate $\mathrm{Fe}$ and $\mathrm{Ru}$ derived bands, indicate that the electronic structure of the Fe-Ru system is that of a coherent alloy as might be anticipated from the similar electronic structures of $\mathrm{BaRu}_{2} \mathrm{As}_{2}$ and $\mathrm{BaFe}_{2} \mathrm{As}_{2}$. In addition, it can be clearly seen that the shape of total DOS near $E_{F}$ is almost unchanged after Ru substitution, except that the values decrease somewhat. This decrease mainly results from the enlarged $d$ band width as the result of increased hopping between transition metals and hybridization involving Ru. $N\left(E_{F}\right)$ is reduced to $3.17 \mathrm{eV}^{-1}$ per $\mathrm{BaFe}_{1.5} \mathrm{Ru}_{0.5} \mathrm{As}_{2}$, as compared to $3.28 \mathrm{eV}^{-1}$ for $\mathrm{BaFe}_{2} \mathrm{As}_{2}$ (note that these GGA values are somewhat larger than the LDA values, e.g. $N\left(E_{F}\right)=3.06$ $\mathrm{eV}^{-1}$ for $\mathrm{BaFe}_{2} \mathrm{As}_{2}$ in Ref. 25). In any case, we can conclude that no additional electron carriers are introduced to $\mathrm{Ru}$ alloyed system in the sense that the band filling is
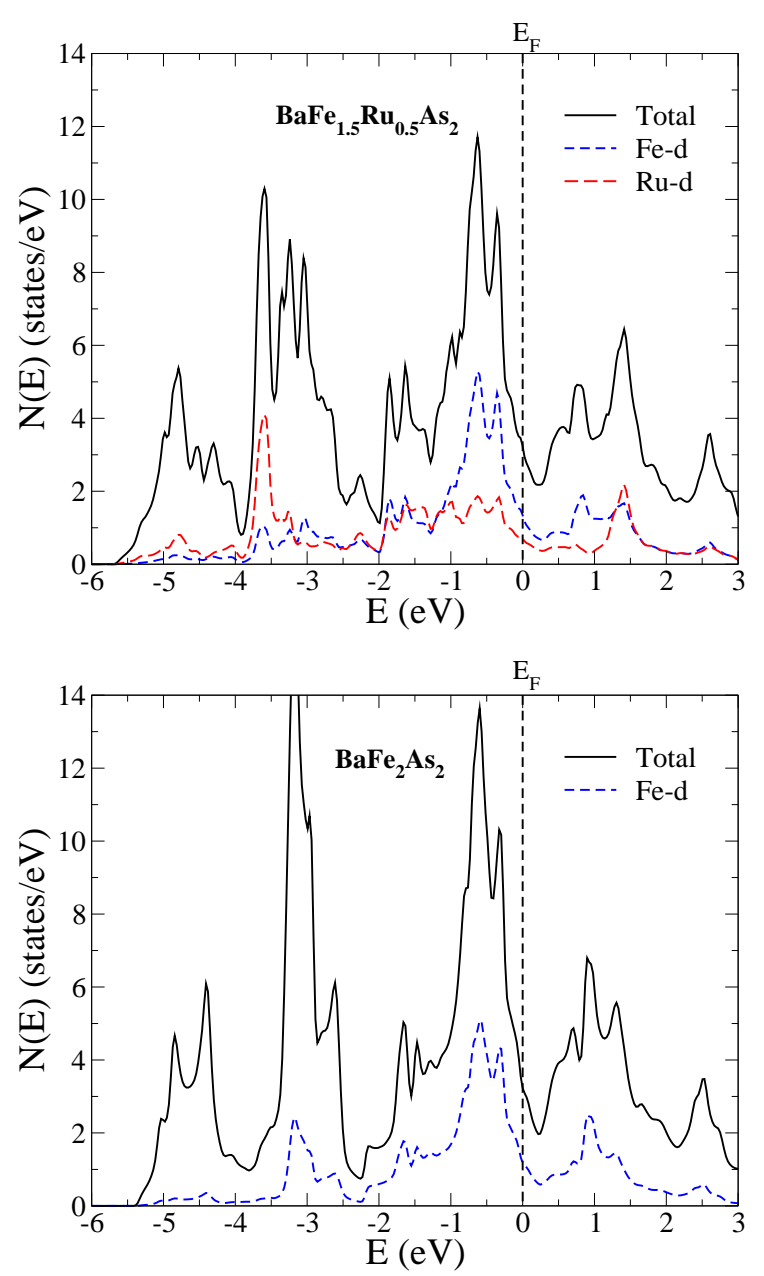

FIG. 3: (Color online) Calculated total DOS and projections for $\mathrm{BaFe}_{1.5} \mathrm{Ru}_{0.5} \mathrm{As}_{2}$ (top), using the quadrupled supercell $A$ (see text). The total DOS is on a per formula $\left(\mathrm{BaFe}_{1.5} \mathrm{Ru}_{0.5} \mathrm{As}_{2}\right)$ basis while the projections are per atom. The total and Fe $d$-projected DOS for $\mathrm{BaFe}_{2} \mathrm{As}_{2}$ (bottom) are presented for comparison.

unchanged.

The general shape of our density of states is simi-

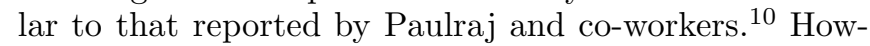
ever, there are significant differences in detail. As mentioned, we find a decrease in the value of $N\left(E_{F}\right)$ upon alloying rather than an increase. We find that for $\mathrm{BaFe}_{1.5} \mathrm{Ru}_{0.5} \mathrm{As}_{2}$ the distribution of $\mathrm{Fe} d$ states is almost the same as in non-spin-polarized $\mathrm{BaFe}_{2} \mathrm{As}_{2}$, giving the main contribution to the states near $E_{F}$ and only modestly mixing with As $p$ states. In contrast, $\mathrm{Ru} d$ derived bands form a peak at $\sim-3.5 \mathrm{eV}$ and overlap in energy with As $p$ states concentrated within the region of $-5.5 \mathrm{eV}$ to $-2.5 \mathrm{eV}$, and thus more strongly hybridized with them in spite of the larger Ru-As distance. This results in a reduced $\mathrm{Ru} d$ contribution to the states near $E_{F}$. Thus the contribution to $N\left(E_{F}\right)$ from Ru $d$ states is lower by half than that of the Fe $d$ states. This plays an important role 


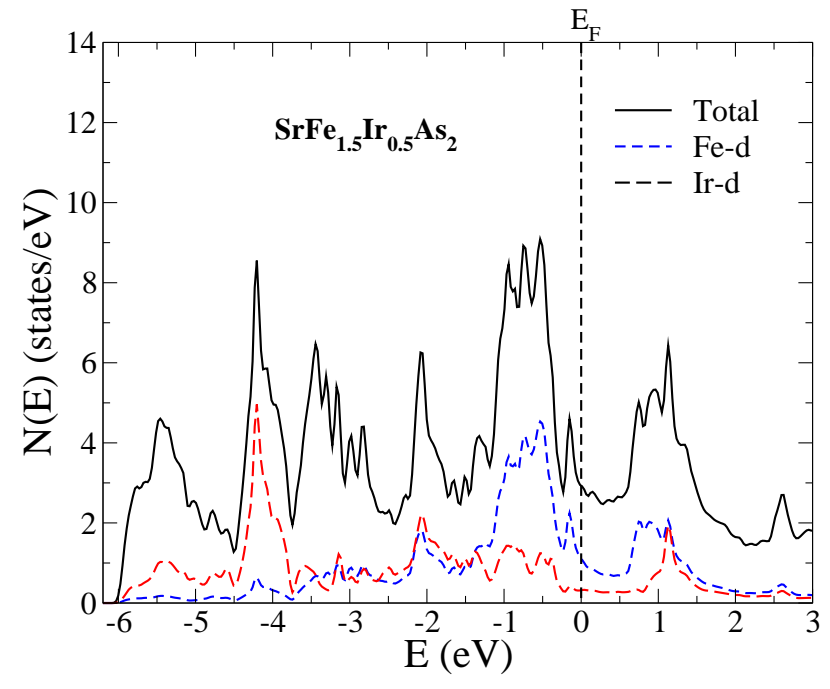

FIG. 4: (Color online) Total and projected electronic DOS of $\mathrm{SrFe}_{1.5} \mathrm{Ir}_{0.5} \mathrm{As}_{2}$, calculated in the same way as that of $\mathrm{BaFe}_{1.5} \mathrm{Ru}_{0.5} \mathrm{As}_{2}$. The tetragonal lattice parameters from experiment (Ref. 15) $a=3.95 \AA$ and $c=12.22 \AA$ were used for supercell simulation.

in the decreased $N\left(E_{F}\right)$ for the $\mathrm{BaFe}_{1-x} \mathrm{Ru}_{x} \mathrm{As}_{2}$ system and is a consequence of Ru-As hybridization.

\section{SUPPRESSION OF THE SDW}

As mentioned, understanding the suppression of the SDW order is important. It is convenient to discuss the magnetic susceptibility, $\chi(\mathbf{q})$, which is given in the random-phase approximation by the enhanced Lindhard susceptibility $\chi(\mathbf{q})=\chi_{0}(\mathbf{q})\left[1-I(\mathbf{q}) \chi_{0}(\mathbf{q})\right]^{-1}$, where $\chi_{0}(\mathbf{q})$ is the bare susceptibility, which is a density of states like term and $I(\mathbf{q})$ is the Stoner parameter, whose $\mathbf{q}$ dependence reflects the changing band character on the Fermi surface. In $\mathrm{BaFe}_{2} \mathrm{As}_{2}$ and other undoped Febased materials, the high $N\left(E_{F}\right) \propto \chi_{0}(0)$ with the large Stoner parameter $I(\sim 0.9 \mathrm{eV}$, characteristic of a $3 d$ transition element, with $N\left(E_{F}\right)$ now on a per spin per Fe basis $)^{26}$ puts them near the Stoner criterion $\left(N\left(E_{F}\right) I>\right.$ 1) for itinerant ferromagnetism magnetism. 3 Further, the strong Fermi nesting between approximately sizematched electron and hole sections yields on top of this a peak in $\chi_{0}(\mathbf{q})$ at the zone corner. This explains the SDW instability at the nesting vector $(1 / 2,1 / 2)$.

Turning to the $\mathrm{Ru}$ alloyed system, on the one hand, more hybridization with As $p$ states and the larger size of the $\mathrm{Ru} 4 d$ orbital relative to the Fe $3 d$ orbital cause a reduction in $N\left(E_{F}\right)$, as mentioned. On the other hand, the atomic-like $I$ is reduced to the value of $\sim 0.6 \mathrm{eV}$ for the mid- $4 d$ transition metal, $\mathrm{Ru}^{26}$ For this coherent alloy system, the Stoner parameter is the average of the atomic Stoner parameters weighted by the squares of the partial contributions of the different $d$ orbitals to the $\chi_{0}$ (i.e. $N\left(E_{F}\right)$ for $\left.\mathbf{q}=0\right) \cdot \frac{26,27}{2}$ The rather smaller $N\left(E_{F}\right)$ of $\mathrm{Ru} d$ states as mentioned would further reduce the contribution from $\mathrm{Ru}$ to the average Stoner parameter. Considering both $I$ and $N\left(E_{F}\right)$ it is clear that the $\mathrm{Ru}$ alloy is much less magnetic than the pure Fe compound. The same factors will apply to the SDW instability, and provide the explanation for its suppression upon $\mathrm{Ru}$ alloying seen both experimentally, and in our direct density functional calculations.

\section{IRIDIUM SUBSTITUTION}

Finally, we also calculated the electronic structure for $5 d$ transition metal, Ir alloyed system, $\mathrm{SrFe}_{1.5} \mathrm{Ir}_{0.5} \mathrm{As}_{2}$, As shown in Fig. 4, the substitution of Fe with Ir also leads to an electronic structure characteristic of a coherent alloy. Compared with $\mathrm{BaFe}_{1.5} \mathrm{Ru}_{0.5} \mathrm{As}_{2}$, the scale of total DOS is further reduced, reflecting still stronger hybridization and expanded band width. This is seen for example in the large Ir contribution to the As $p$ derived bands at $\sim-4 \mathrm{eV}$ and the correspondingly reduced Ir contribution to the metal $d$ bands from $\sim-2 \mathrm{eV}$ to 2 $\mathrm{eV}$ relative to $E_{F}$. More importantly, $E_{F}$ clearly shifts upwards towards the bottom of the pseudogap, which reflects additional electrons that are doped into the $d$ bands by alloying this Co column transition metal. The doping amounts to one carrier per Ir since, as for $\mathrm{Ru}$, the electronic structure is coherent and no additional bands are introduced. The value of $N\left(E_{F}\right)$ is $2.91 \mathrm{eV}^{-1}$ per formula of $\mathrm{SrFe}_{1.5} \mathrm{Ir}_{0.5} \mathrm{As}_{2}$. In addition, we note that the Stoner parameter of Ir is rather low, $I_{\mathrm{Ir}} \sim 0.5 \mathrm{eV} .26$ Thus, the suppression of SDW order in this Ir-alloyed system ${ }^{15}$ may be attributed to effects of both a decreased Stoner factor and electron doping.

\section{SUMMARY AND CONCLUSIONS}

In summary, we show based on density functional calculations the alloying $\mathrm{BaFe}_{2} \mathrm{As}_{2}$ with $\mathrm{Ru}$ and $\mathrm{SrFe}_{2} \mathrm{As}_{2}$ with Ir results in the formation of a coherent electronic structure without additional localized states associated with the impurity atoms. As such, the suppression of SDW magnetic order for the $\mathrm{Ru}$ alloyed $\mathrm{Ba}(\mathrm{Sr}) \mathrm{Fe}_{2} \mathrm{As}_{2}$ system is due to the increased hybridization and a reduced average Stoner parameter reflecting the different chemistry of $3 d$ and $4 d$ transition elements. A similar consideration applies to alloying with Ir, although in that system Ir also provides doping. We note that scattering due to $\mathrm{Ru} / \mathrm{Ir}$ disorder in the actual alloys might also play a role in suppressing the SDW, since scattering generally works against a nesting induced instability. Also it should be mentioned that the detailed role of $4 d$ and $5 d$ transition metals on the interplay between magnetism and superconductivity in Fe-based superconductors might be quite complex. For example, $\mathrm{Ru}$ alloying is found to completely suppress SDW order in the $\mathrm{PrFe}_{1-x} \mathrm{Ru}_{x} \mathrm{AsO}$ system but no superconductivity 
emerges, at least in samples investigated to date ${ }^{28}$ However, Ir doped $\mathrm{LaFe}_{1-x} \mathrm{Ir}_{x} \mathrm{AsO}$ is reported to show superconductivity with $T_{c}$ up to $10.5 \mathrm{~K} .29$ Further studies will be helpful in understanding these differences. In any case, substitution of $\mathrm{Fe}$ with $4 d$ and $5 d$ transition metals in Fe-based materials provides another avenue for suppressing SDW magnetic order and thereby inducing superconductivity in Fe-based materials, even in the absence of doping.

\section{Acknowledgments}

We are grateful for helpful discussions and assistance from A. Subedi. This work was supported by the Department of Energy, Division of Materials Sciences and Engineering.
1 Y. Kamihara, T. Watanabe, M. Hirano, and H. Hosono, J. Am. Chem. Soc 130, 3296 (2008).

2 C. de la Cruz, Q. Huang, J. W. Lynn, J. Li, W. Ratcliff II, J. L. Zarestky, H. A. Mook, G. F. Chen, J. L. Luo, N. L. Wang, et al., Nature 453, 899 (2008).

3 D. J. Singh and M. H. Du, Phys. Rev. Lett. 100, 237003 (2008).

4 I. I. Mazin, D. J. Singh, M. D. Johannes, and M. H. Du, Phys. Rev. Lett. 101, 057003 (2008).

5 J. Dong, H. J. Zhang, G. Xu, Z. Li, G. Li, W. Z. Hu, D. Wu, G. F. Chen, X. Dai, J. L. Luo, et al., Europhys. Lett. 83, 27006 (2008).

${ }^{6}$ G. F. Chen, Z. Li, D. Wu, G. Li, W. Z. Hu, J. Dong, P. Zheng, J. L. Luo, and N. L. Wang, Phys. Rev. Lett. 100, 247002 (2008).

7 P. L. Alireza, Y. T. C. Ko, J. Gillett, C. M. Petrone, J. M. Cole, G. G. Lonzarich, and S. E. Sebastian, J. Phys. Condens. Matter 21, 012208 (2009).

8 A. S. Sefat, R. Jin, M. A. McGuire, B. C. Sales, D. J. Singh, and D. Mandrus, Phys. Rev. Lett. 101, 117004 (2008).

${ }^{9}$ L. Li, Y. Luo, Q. Wang, H. Chen, Z. Ren, Q. Tao, Y. Li, X. Lin, M. He, Z. Zhu, et al., New J. Phys. 11, 025008 (2009).

10 S. Paulraj, S. Sharma, A. Bharathi, A. T. Satya, S. Chandra, Y. Hariharan, and C. S. Sundar, arXiv:0902.2728 (2009).

11 W. Schnelle, A. Leithe-Jasper, R. Gumeniuk, U. Burkhardt, D. Kasinathan, and H. Rosner, arXiv:0903.4668 (2009).

12 Y. Qi, L. Wang, Z. Gao, D. Wang, X. Zhang, and Y. Ma, arXiv:0903.4967 (2009).

${ }^{13}$ F. Han, X. Zhu, P. Cheng, B. Shen, and H. H. Wen, arXiv:0903.1028 (2009).

14 X. Zhu, F. Han, P. Cheng, B. Shen, and H. H. Wen,
arXiv:0903.0323 (2009).

15 F. Han, X. Zhu, Y. Jia, L. Fang, P. Cheng, H. Luo, B. Shen, and H.-H. Wen, arXiv:0902.3957 (2009).

16 R. Nath, Y. Singh, and D. C. Johnston, arXiv:0901.4582 (2009).

17 J. P. Perdew, K. Burke, and M. Ernzerhof, Phys. Rev. Lett. 77, 3865 (1996).

18 P. Blaha, K. Schwarz, G. Madsen, D. Kvasnicka, and J. Luitz, An Augmented Plane Wave+ Local Orbitals Program for Calculating Crystal Properties (K. Schwarz, Tech. Univ. Wien, Austria) (2001).

19 E. Sjostedt, L. Nordstrom, and D. J. Singh, Solid State Commun. 114, 15 (2000).

20 D. J. Singh and L. Nordstrom, Planewaves Pseudopotentials and the LAPW Method, 2nd Ed. (Springer, Berlin, 2006).

21 W. Jeitschko, R. Glaum, and L. Boonk, J. Solid State Chem. 69, 93 (1987).

22 A. S. Sefat, D. J. Singh, R. Jin, M. A. McGuire, B. C. Sales, and D. Mandrus, Phys. Rev. B 79, 024512 (2009).

23 A. Subedi and D. J. Singh, Phys. Rev. B 78, 132511 (2008).

24 M. Rotter, M. Tegel, D. Johrendt, I. Schellenberg, W. Hermes, and R. Pöttgen, Phys. Rev. B 78, 020503(R) (2008).

25 D. J. Singh, Phys. Rev. B 78, 094511 (2008).

${ }^{26}$ O. K. Andersen, J. Madsen, U. K. Poulsen, O. Jepsen, and J. Kollar, Physica B+C 86, 249 (1977).

27 I. I. Mazin and D. J. Singh, J. Phys. Chem. Solids 59, 2185 (1998).

28 M. A. McGuire, D. J. Singh, A. S. Sefat, B. C. Sales, and D. Mandrus, arXiv:0904.1570 (2009).

29 Y. Qi, L. Wang, Z. Gao, D. Wang, X. Zhang, and Y. Ma, arXiv:0904.0772 (2009). 